\title{
Changing the antibiotic prescribing behaviour of clinicians in primary care
}

\author{
Sarah Tonkin-Crine PhD, Kyle Knox MBChB
}

See also page 108 and www.cmaj.ca/lookup/doi/10.1503/cmaj.150601

Competing interests: Sarah Tonkin-Crine is funded by the UK National Institute of Health Research (NIHR) Health Protection Research Unit in Healthcare Associated Infections and Antimicrobial Resistance, hosted by the University of Oxford. Kyle Knox has received grants from the NIHR under the Oxford Biomedical Research Centre Infection Theme.

This article was solicited and has not been peer reviewed.

Correspondence to: Kyle Knox,

kyle.knox@phc.ox.ac.uk

CMAJ 2016. DOI:10.1503 /cmaj.151103
1 ntimicrobial resistance has been comquences of inaction would seem apocalyptic: by 2050, it is estimated that 10 million lives will be lost annually because of untreatable infections, at a cumulative global cost of US $\$ 100$ trillion. ${ }^{1}$ The scale of the problem will require a range of approaches, and addressing the unnecessary use of antibiotics is one priority.

Overuse of antibiotics is one of the main drivers of the increase in resistance, yet between 2000 and 2010, the global consumption of antibiotics increased by $36 \% .^{2}$ With only five new classes of antibiotics discovered since the 1970s, the emphasis in addressing resistance has been on the conservation of current antibiotics.

In a linked study, Vellinga and colleagues ${ }^{3}$ investigated an intervention to increase the proportion of first-line antibiotics prescribed for patients with urinary tract infection presenting to Irish general practices. ${ }^{4}$ They had previously identified that less than $40 \%$ of antibiotic prescriptions for this type of infection in Ireland were for recommended first-line antibiotics. ${ }^{4}$

Clinicians can play an important role in reducing resistance through prudent prescribing. This approach includes deciding when a bacterial infection is likely and then choosing the correct antibiotic, route and dose. Antimicrobial policies help clinicians to make these decisions, and antimicrobial stewardship programs use interventions to encourage behaviours that pro-

\section{KEY POINTS}

- Primary care has a key role in acting to reduce antibiotic resistance.

- Interventions targeted toward general practitioners to support appropriate antibiotic prescribing can have positive effects on first-line prescribing.

- Complex interventions may have unintended effects on clinicians' behaviour, such as an increase in overall antibiotic prescribing.

- Measuring mediators of behaviour change, informed by theory, can specify how an intervention works and explain unintended consequences of complex interventions. mote evidence-based practice, mostly focused on reducing the prescribing of antibiotics. These interventions incorporate many components, such as audit and feedback, clinician or patient education, and use of point-of-care tests.

In Europe, overall outpatient antibiotic use is increasing, ${ }^{5}$ and urinary tract infections represent one of the most common indications. This type of infection is well suited to interventions that target antibiotic use, because both the causal bacteria and their resistance profiles are predictable.

Vellinga and colleagues designed their multifaceted intervention to change clinicians' prescribing behaviour. The intervention contained educational workshops with audit and feedback, elements that have previously been shown to be effective and acceptable to general practitioners. ${ }^{6}$ They selected these components to fit with Irish general practice so as to create an intervention that was feasible and self-sustaining.

The investigators used a three-arm cluster randomized design, which is commonly used in intervention studies to minimize unmeasured potential biases, such as high- or low-prescribing cultures. The observed absence of differences in baseline prescribing data among the three arms suggests that randomization was effective, and the study population appeared representative of patients presenting to general practices with urinary tract infection (mean age 51-56 yr; $88 \%-92 \%$ women). ${ }^{7}$

The sample size was based on a $10 \%$ increase in first-line antimicrobial prescriptions in the intervention arms, which was consistent with reported effects. ${ }^{8}$ The increases in first-line antibiotic prescriptions of $23 \%$ and $17 \%$ in intervention arms A and B, respectively — with a secondary analysis that used prescribing of nitrofurantoin as the outcome showing an even larger effect indicated that the intervention was successful. The reduction in repeat consultations (a proxy for treatment failure and thus antibiotic resistance) among patients who received nitrofurantoin, but not in the intervention arms overall, suggests that 
improving adherence to prescribing guidelines leads to better patient outcomes.

Although these results are promising, the size of the effects can depend on study design ${ }^{9,10}$ and may not be replicated in the unselected population of clinical practice. In addition, the effect of delayed prescribing, the only difference between the two intervention arms, was measured indirectly. Therefore, the effect of education about delayed prescribing on general practitioners' actual prescribing is unclear.

An unexpected (and unexplained) consequence of the intervention was an increase in the overall prescribing of antibiotics in the intervention arms relative to the control arm, but perhaps this points to how interventions change prescribing behaviour.

To understand how a behavioural intervention works, it is important to measure the potential mediators of change, which can be identified from theories of behaviour. Interventions that are successful in changing knowledge and attitudes often change intentions to prescribe, which, in an environment that supports nonprescription, can lead to a change in prescribing behaviour. Understanding the change in general practitioners' knowledge or attitudes could help to explain changes in first-line prescribing and could indicate why prescribing increased overall.

In this study, general practitioners in both intervention arms were encouraged to prescribe a first-line antibiotic. Those in arm B were also prompted to delay the prescription. With current rhetoric that focuses more on avoiding the prescribing of antibiotics, this intervention may have given general practitioners "permission" to prescribe, which may in turn have contributed to the unexpected increase in overall antibiotic prescribing. An explicit link to theory and more detail about the exact messages provided could offer an explanation for the unintended effects.

What are the implications for primary care practice? A theory-based intervention should de- tail exactly how its content is seeking to change specific influences on behaviour. By considering possible unintended consequences of interventions during development of the intervention, undesirable behaviours, such as increased prescribing, could be avoided. Nevertheless, this paper offers encouragement that general practitioners can make a meaningful contribution to preserving the limited antibiotics available today and that they have a part to play in the global approach to this crisis.

\section{References}

1. O'Neill J. Review on antimicrobial resistance. Antimicrobial resistance: tackling a crisis for the health and wealth of nations. London: Wellcome Trust; 2014.

2. Van Boeckel TP, Gandra S, Ashok A, et al. Global antibiotic consumption 2000 to 2010: an analysis of national pharmaceutical sales data. Lancet Infect Dis 2014;14:742-50.

3. Vellinga A, Galvin S, Duane S, et al. Intervention to improve the quality of antimicrobial prescribing for urinary tract infection: a cluster randomized trial. CMAJ 2016;188:108-15.

4. Duane S, Callan A, Galvin S, et al. Supporting the improvement and management of prescribing for urinary tract infections (SIMPle): protocol for a cluster randomised trial. Trials 2013;14:441.

5. Adriaenssens N, Coenen S, Versporten A, et al.; ESAC Project Group. European Surveillance of Antimicrobial Consumption (ESAC): outpatient antibiotic use in Europe (1997-2009). J Antimicrob Chemother 2011;66 Suppl 6:vi3-12.

6. Tonkin-Crine S, Yardley L, Coenen S, et al. GPs' views in five European countries of interventions to promote prudent antibiotic use. Br J Gen Pract 2011;61:e252-61.

7. Foxman B. The epidemiology of urinary tract infection. Nat Rev Urol 2010;7:653-60.

8. Ranji SR, Steinman M, Shojania K, et al. Interventions to reduce unnecessary antibiotic prescribing. A systematic review and quantitative analysis. Med Care 2008;46:847-62.

9. Butler CC, Simpson SA, Dunstan F, et al. Effectiveness of multifaceted educational programme to reduce antibiotic dispensing in primary care: practice based randomised controlled trial. BMJ 2012;344:d8173.

10. Little P, Stuart B, Francis N, et al.; GRACE Consortium. Effects of internet-based training on antibiotic prescribing rates for acute respiratory-tract infections: a multinational, cluster, randomised, factorial, controlled trial. Lancet 2013; $382: 1175-82$

Affiliation: Nuffield Department of Primary Care Health Sciences, University of Oxford, Oxford, United Kingdom

Contributors: Both authors contributed to the conception and writing of the article, approved the final version to be published and agreed to act as guarantors of the work. 